\title{
Optimization and Analysis of Structure about Lifting Device of Logistics Sorting
}

\author{
G. ZHAO ${ }^{1}$, Zs. TIBA ${ }^{2}$, J. MENYHART ${ }^{3}$ \\ ${ }^{1}$ University of Science and Technology LiaoNing, Liaoning Anshan, 114051, China, Engineering Automation, \\ 821116807@qq.com \\ ${ }^{2}$ University of Debrecen, Faculty of Engineering, Institute of Mechanical and Vehicle Engineering, Department of \\ Air- and Road Vehicles, tiba@eng.unideb.hu \\ ${ }^{3}$ University of Debrecen, Faculty of Engineering, Institute of Mechanical and Vehicle Engineering, Department of \\ Air- and Road Vehicles, jozsef.menyhart@eng.unideb.hu
}

Abstract. the lifting device of a logistics sorting machine needs high frequency upward and downward reciprocating motion therefore the cutting fork arms and matching parts of the shaft are often worn out. In this paper the problem of the shear fork types applied for the lifting mechanism is studied at first. Then the advanced numerical simulation software ANSYS adopted for the lifting mechanism of the shear fork type, and the means of virtual simulation is introduced. Hence the possible location of faults and fault modes are analysed. Then improving measures about the lifting mechanism of the logistics sorting machine are suggested.

\section{Introduction}

The function of a lifting device in the system of logistics sorting machines is to lift and deliver the product package to the transporting organs or to a venue from where the sorted package is transferred by transporting organs to their destination. Its operational characteristics are as follows: it needs to do high frequency upward and downward moreover downward movements of which most of them are load unit movements. As the lift needs high frequency to upward and downward reciprocating movements a wear phenomenon often occurs in the matching part of the hole of the shear fork arm and the shaft. Long holes will occur at the matching hole of the shear fork arm and where the shaft connection exists for a long time. This will affect its service life [3]. The existing lifting device is decomposed. After disassembly and measurement, the drawings of the lifting device of the logistics sorting machine are drawn. The ANSYS numerical simulation parameterized model of connecting rods with different angles is established. The stress characteristics of the lifting device of the logistics sorting machine are obtained through force analysis and its possible failure location and failure mode occur. According to the results of relevant research and numerical simulation analyses, the improvement measures of the lifting device of logistics sorting machine are suggested. 


\section{Analytical Principles}

The existing lifting device is decomposed and the lifting device drawings of the logistics sorting machine are drawn after disassembly and measurement. The motor's rotation is driven by worm gear and worm mechanism is provided by belt. Thus upward and downward reciprocating motion of the lifter is realized and transformation from motor's rotation to linear motion is completed. The lifting device of the sorting machine is a typical shear fork mechanism.

Generally, there seem to be two driving modes of shear fork lifts: one is driven by hydraulic cylinder and the other is driven by a worm wheel and a worm. A worm wheel and a worm drive mode have the advantages of simple structure, good stability, long service life and low accident rate. Therefore, a worm wheel and a worm drive mode are used in the lifting device of the logistics sorting machine [4][5].

Each shear rod of the fork-cutting mechanism can be considered as connected elements with the two hinged sections at one end and a fixed beam element at the other end. The shear fork mechanism is the main body of the shear fork lifting platform and the main bearing member.

Taking a single-stage scissors lift as an example [see Fig. 1], the horizontal velocity of slide B and the vertical velocity of elevator ED are analysed. The mechanism consists of five rigid bodies, $N=5$, and the base point of the common base is point $A$. The coordinates of the system are $n=3 N=15$. In the figure the cutting fork arm BE is B1, the cutting fork arm AD is B2, the slider B is B3, the slider D is B4, and the rod ED is B5. There are four rotating hinges and two sliding hinges in the system. The absolute position constraints in $\mathrm{X}$ and $\mathrm{Y}$ directions are found at the end of $\mathrm{A}$. So the number of principal constraints of the whole system $S=14$,

Freedom $\delta=15-14=1[6]$

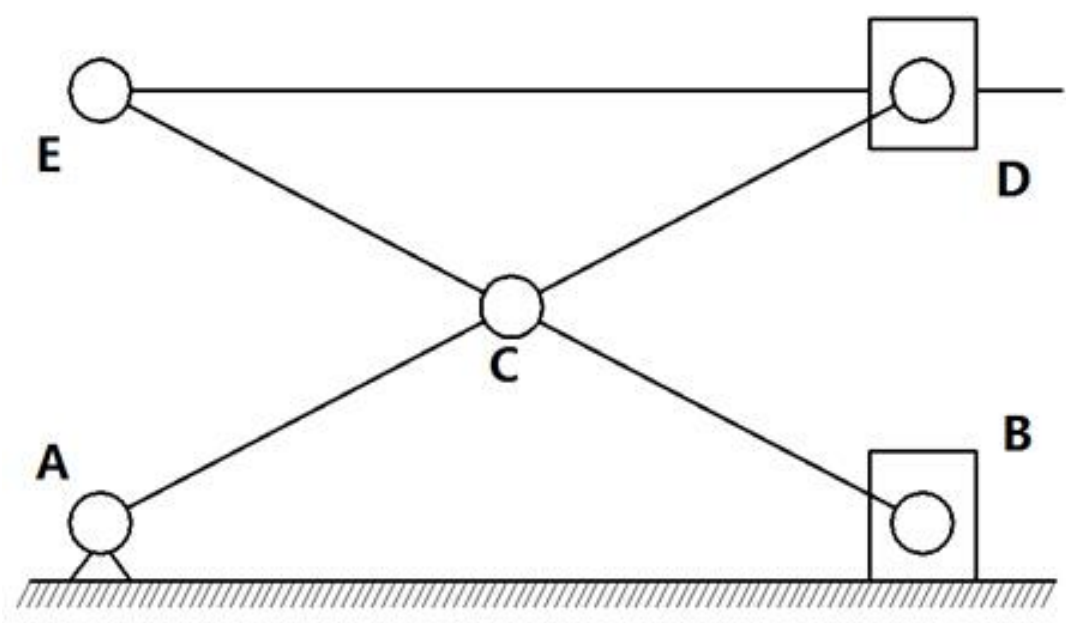

Fig.1 Simplified kinematic model of scissors

In order to get more intuitive motion characteristics of the fork-cutting mechanism, the threedimensional solid model of the first-order fork-cutting mechanism was established by the School of Automotive Engineering of Wuhan University of Technology with ADMAS software, moreover motion 
simulation was carried out. The velocity curve of the platform during the descending process of the first-order fork-cutting mechanism was obtained [see Figure 2, 3]. On the basis of the analysis results it is seen that the fork-cutting mechanism is working. At first it has the characteristics of being fast and then it slows down. It can make the lifting device slow and then it can accelerate in the process of laying down heavy objects and can play a buffer role.

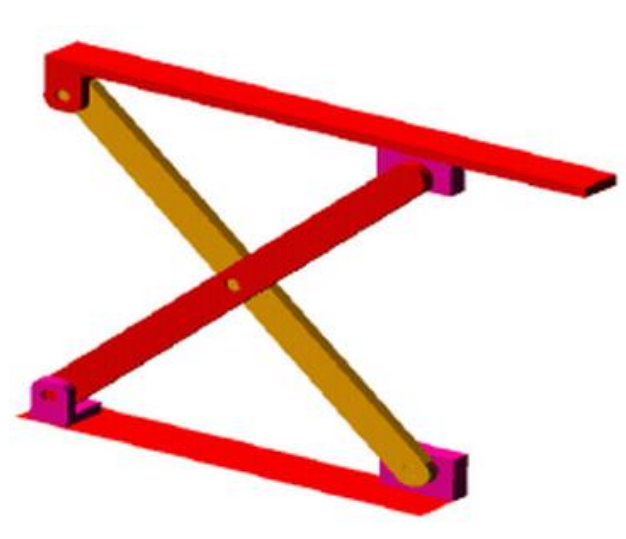

Fig.2 Three-dimensional model of ADMAS simulation

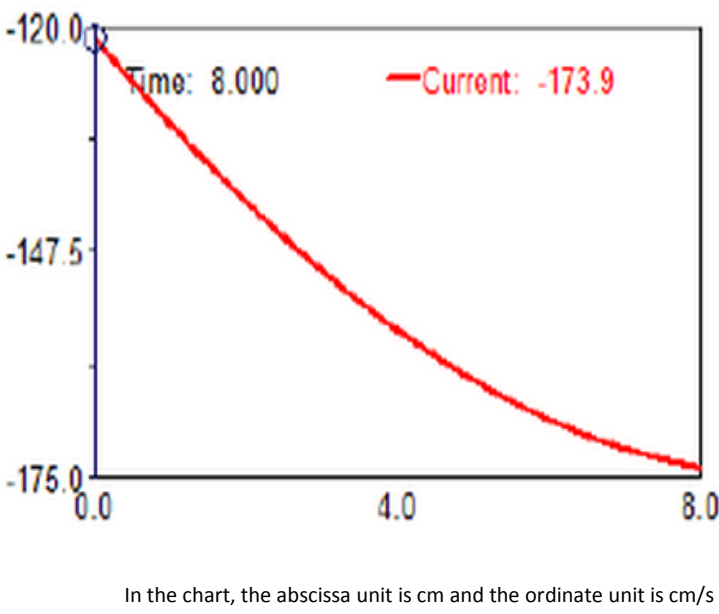

Fig.3 Y-Direction Velocity Curve of Platform

\section{Damage Analysis of Scissors}

Random fatigue load accompanied by occasional sudden load is the main form of loading on the shear fork mechanism. After bearing the stress distribution on the structure of the shear fork mechanism it becomes more complex and its damage situation varies with the structural form. However, according to its bearing characteristics there are two main damage situations: [1] forced damage when it is suddenly overloaded. This situation generally does not occur under normal working conditions only when the shear fork lifting platform is suddenly impacted and the shear fork arm may be damaged by sudden overload; [2] fatigue damage caused by random fluctuating pressure load. The damage of the shear fork mechanism is far below the breaking pressure load which is the main situation of the damage of the shear fork mechanism at present [7]. Most of the shear fork mechanism is due to crack initiation and gradual propagation in some stress sensitive parts under fatigue load which eventually leads to brittle fracture. And fatigue load makes the shear fork mechanism constantly suffer friction and abrasion in the use process which will also promote the initial crack initiation and accelerate crack propagation. Therefore, the final failure of the shearing mechanism is actually the result of the interaction of many factors. After observing used shear fork mechanism, the wearing part is mainly at the pin hole of the shear fork arm, which is the worse part in use. It is mainly caused by the contact, extrusion and relative rotation between the pin and the shear fork arm. The longer the use time of the shear fork mechanism is the worse the wear will be. 


\section{Numerical Simulation and Analysis}

Advanced three-dimensional finite element simulation software ANSYS [8] is used aiming at analysing the usage and damage characteristics of the shear fork mechanism of lifting device. The shear fork arm of the main load-bearing part in the shear fork mechanism is simulated and analysed, and the stress distribution law of the shear fork arm under the working state is simulated.

\subsection{Establishment of three-dimensional simulation model}

According to Professors Liu Junyi and Yang Gang of PLA University of Science and Technology, the stress value of the scissor arm is the largest when the angle between the scissors is the smallest [scissors mechanism]. In order to ensure the accuracy and representativeness of the calculation and save calculation time only this analysis is implemented. The force and deformation of a scissor arm at the minimum working angle are calculated. From figs. 4 and 5, it can be seen that the axial stress of each section before and after simplification [the numerical value of the axial stress in the figure] has changed and the position of the maximum axial stress has changed. As the simulation results are close to calculation results the maximum axial pressure of the three-stage shear fork mechanism can be estimated approximately by the simplified calculation results. In addition, the maximum axial tension and compression pressure of the two mechanisms decrease nonlinearly with the increase of theta. Furthermore, the values are far less than the allowable values of the axial stress. After calculation the minimum working angle of the shear fork mechanism in the lifting device of the current logistics sorting machine is 7.36 degrees. Therefore, this numerical simulation analysis chooses the working angle of 7.36 degrees [the three-dimensional model established is shown in Fig. 6].
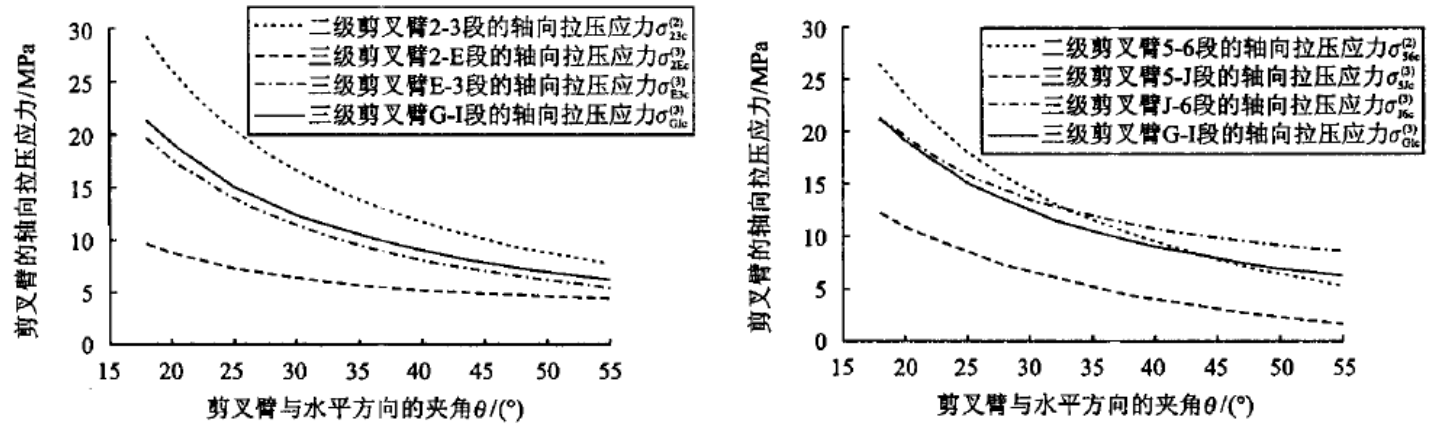

Fig.4 The Curve of Relation Between Tensile Value Force and Angle Fig.5 Curve of relationship between compressive stress and angle 


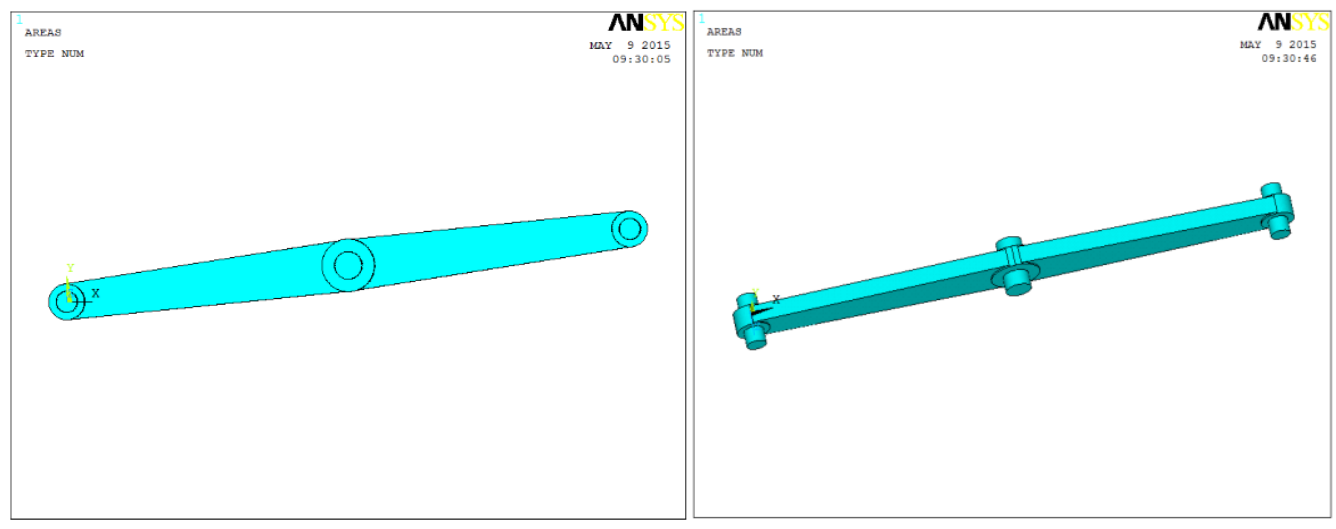

Fig.6 Three-dimensional finite element model for numerical simulation analysis

\subsection{Parameter determination, meshing, contact pair definition and load input}

At present the material of a shear fork arm in a lifting device applied as a sorting machine in logistics is medium carbon cast steel ZG 270-500 with certain self-lubrication function. ZG 270-500 has certain toughness, plasticity, strength and hardness. It is widely used in flywheel, vehicle coupler, bearing seat, shear fork arm, mobile worktable, etc. The chemical composition of ZG 270-500 is shown in Table 1. The mechanical properties of ZG $270-500$ are yield limit $[\geq 270 \mathrm{MPa}$ ], tensile strength [ $\geq 500 \mathrm{MPa}$ ], elastic modulus [> $2.05 \mathrm{e} 11 \mathrm{~N} / \mathrm{m}]$ and density [> $7820 \mathrm{~kg} / \mathrm{m} 3]$.

\begin{tabular}{|c|c|c|c|c|c|c|c|c|c|c|}
\hline $\begin{array}{c}\text { Chemical } \\
\text { composition }\end{array}$ & C & Si & Mn & S & P & Ni & Cr & Cu & Mo & V \\
\hline Proportion (\%) & 0.4 & 0.5 & 0.9 & 0.04 & 0.04 & 0.3 & 0.35 & 0.30 & 0.20 & 0.05 \\
\hline
\end{tabular}

Table 1. Proportion of chemical constituents in ZG270-500

The hole of the shear fork arm in the shear fork mechanism of a lifting device contacts with the connecting shaft face-to-face. Because the material of the connecting shaft is $45 \#$ steel, the hardness and wear resistance of the connecting shaft are better than ZG 270-500. Therefore, in this analysis the connecting shaft is set as a rigid body, the surface of the connecting shaft is set as the contact surface and the inner surface of the hole of the shear fork arm is set as the target. Target 170 and CONTA173 with contact analysis function are selected as the meshing element types of connection shaft and scissor arm respectively. The solid model is meshed and the contact pairs are defined respectively on the inner surface of the three holes of scissor arm and the corresponding connecting axis. According to the field investigation the rated load of the lifting device of the logistics sorting machine is $500 \mathrm{~kg}$, the lifting time is 1 second, and the load parameters are input.

\subsection{Analysis of calculation results}

As the results of the above analysis it can be concluded that the maximum stress of the shear fork arm under the maximum rated load is $61.72 \mathrm{MPa}$. This is far below the yield limit of $270 \mathrm{MPa}$. Therefore, the strength of the shear fork arm of the lifting device can meet the working requirements. On the basis of the stress distribution law it can be seen that the maximum stress position of the shear fork 
arm appears above the middle hole of the shear fork arm, and the stress value of the two sides of the hole is obviously larger than that of the surrounding part.

\subsection{Improvement measures}

The pinhole of the shear fork arm of the shear fork mechanism in the lifting device of the sorting machine applied in logistics produces greater stress when it works. This is the easily damaged part in use and wear is more serious when it is used for a long time. In addition, the wear caused by the contact, extrusion and relative rotation between the pin and the shear fork arm is more serious when the shear fork mechanism is used for a long time. When the shear fork mechanism is loaded there seems to be a large stress value in the middle pinhole of the shear fork arm and its surrounding parts, where most of the shear fork mechanisms are destroyed. Therefore, wear and load-bearing degree will have a great impact on the strength and durability of the area. Thus, it is suggested to install bearings in the three holes of the shear fork arm of the shear fork mechanism in the lifting device of the sorting machine applied in logistics. The specific measures are as follows: enlarging the three holes of the connecting rod [enlarging to $22 \mathrm{~mm}$ ], leaving a $2 \mathrm{~mm}$ stop at the back of the hole and processing the front end of the hole to install the ring groove of the spring stop washer for fixing bearings, and selecting $\mathrm{N}$ as the bearing. It is AV4002 $407410215^{*} 22^{*} 17$ needle roller bearing.

\section{Conclusion}

This paper examines the existing lifting device of a sorting machine applied in logistics. By comparing the simulation results with the theoretical values, the practicability and rationality of simplification and improvement measures are introduced. Bearings are installed in the three holes of the shear fork arm of the shear fork mechanism in the lifting device of the sorting machine applied in logistics to complete transformation from motor rotation to linear motion. However, in this paper the level of the research and the discussion on lifting device of the sorting machine applied in logistics is still relatively basic. There seems to be no more systematic analyses on the mechanical function of threelevel scissors. I will prepare a more systematic research and analysis in my future work.

\section{References}

[1] Liu.J.Y.,Yang.G.,Zhang.W.J. Analysis of force characteristics of scissor type hoist. Journal of PLA University of science and technology.2014.04 (2): 134-138

[2] Zhao.M.J.,Liu.J.X.,Chang.A.M.,Li.J. Force analysis of scissor lift. New technology and process.2013.0 (3):18-20

[3] Baidu Library. Dynamic analysis of scissor type lifting mechanism

[4] Shi.X.Y.,Yu.B.F., Test method for reliability of scissor lift. Hoisting and transportation machinery,2013 (5):75-77

[5] Zhang.Y.C.,Zhao.J.Y.,Lu.N., Scissor type hydraulic lifting platform. Hydraulic and pneumatic,2011 11:71-73

[6] Pu.L.G.,Ji.M.G., Machine Design 7.Beijing Higher Education Press 2001 
International Journal of Engineering and Management Sciences (IJEMS) Vol. 4. (2019). No. 4

DOI: 10.21791/IJEMS.2019.4.49.

[7] HU.X.Z.,Hu.J.K.,He.A.H., Modeling of scissor type lifting mechanism and study of pipe parameters, Mechanical research and Application,2006,19(4):84-85

[8] Li.S.Y.,Li.G.Y., ANSYS9.0 Foundation and application examples. Beijing: China Science and Culture Press, 2005 